\title{
Low Birthweight and Premature Birth Are Risk Factors for Podocytopenia and Focal Segmental Glomerulosclerosis
}

\author{
Yohei Ikezumi ${ }^{a}$ Toshiaki Suzuki ${ }^{a}$ Tamaki Karasawa ${ }^{a}$ Takeshi Yamada ${ }^{a}$ \\ Hiroya Hasegawa $^{a}$ Hiroko Nishimura $^{b}$ Makoto Uchiyama $^{a}$ \\ ${ }^{a}$ Department of Pediatrics, Niigata University Medical and Dental Hospital, and ${ }^{\mathrm{b}}$ Institute of Nephrology, \\ Niigata University Graduate School of Medical and Dental Sciences, Niigata, Japan
}

\section{Key Words}

Focal segmental glomerulosclerosis · Low birthweight .

Premature birth · Podocyte

\begin{abstract}
Background: Recent reports suggest that low birthweight (LBW) is a risk factor for kidney diseases, including focal segmental glomerulosclerosis (FSGS), although the underlying pathological mechanism remains unknown. Podocyte loss triggers glomerulosclerosis; however, whether FSGS in LBW children is associated with podocytopenia is unclear. Methods: We reviewed the birthweights and gestational age of all patients who underwent renal biopsies from 1995 to 2011 at our Institute. Sixteen patients had FSGS, of which 6 (37.5\%) had LBW; this LBW rate was significantly higher than the overall LBW rate in Japan (9.7\%). The incidence of LBW was also high in patients with minimal change nephrotic syndrome (MCNS; $12.5 \%)$. The glomerular cell numbers in biopsy sections were calculated using computer image analysis and compared with FSGS of normal birthweight (NBWFSGS). Biopsy specimens from age-matched patients with MCNS were also compared. Wilms' tumor-1 (WT1) immunohistochemistry was performed to enumerate the podocytes. Results: All patients in the LBW-FSGS group were also pre-
\end{abstract}

term, with an average gestational age of 25.8 weeks. The number of podocytes per glomerulus in the LBW-FSGS patients was 34 and $24 \%$ lower as compared to that in the MCNS patients $(p<0.01)$ and the NBW-FSGS patients $(p<$ $0.05)$, respectively. Similar results were observed for the WT1-positive glomerular cell number. Conclusion: LBW and premature birth were associated with FSGS development. The possibility that LBW and premature birth may be predisposing factors for severe podocytopenia in children with FSGS warrants further investigation.

Copyright @ 2013 S. Karger AG, Basel

\section{Introduction}

Recent studies have revealed that fetal growth influences chronic disease in later life [1]. This hypothesis, which is called the Developmental Origins of Health and Disease, proposes that the physiological adaptations of the fetus in utero in response to a deleterious milieu increase the susceptibility of the individual to many chronic diseases, including metabolic syndrome, diabetes, hypertension, cardiovascular disease, and chronic kidney disease [1-4]. Furthermore, it is hypothesized that babies born with a lower number of nephrons due to intrauter-

\section{KARGER}

E-Mail karger@karger.com

www.karger.com/ajn
(C) 2013 S. Karger AG, Basel

0250-8095/13/0382-0149\$38.00/0
Yohei Ikezumi, MD, PhD

Department of Pediatrics

Niigata University Medical and Dental Hospital

Asahimachi-dori, Niigata 951-8510 (Japan)

E-Mail ikezumi@med.niigata-u.ac.jp 
ine growth retardation are susceptible to various types of kidney injuries that lead to progressive kidney dysfunction and glomerulosclerosis $[5,6]$. Moreover, it has been reported that low birthweight (LBW) promotes the development of glomerular alterations such as mesangial matrix expansion and hyalinosis, and that it could be a cause of focal segmental glomerulosclerosis (FSGS) [7], although the underlying pathological mechanism has not been studied.

It is now generally accepted that the loss of podocytes triggers glomerulosclerosis [8-10]. Moreover, almost any glomerular disease that ultimately leads to end-stage renal failure triggers the formation of FSGS lesions, which are one of the most common forms of glomerular pathologies and are either primary or secondary in nature [11]. Primary FSGS is often attributed to genetic mutations of podocyte-related genes, while secondary FSGS is associated with chronic glomerulonephritis, infections, hypertension, obesity, drugs as well as LBW. However, the mechanisms underlying the development of FSGS lesions in LBW children have not been fully elucidated. Therefore, we histologically analyzed the number of glomerular podocytes in children with FSGS, in addition to the prevalence of LBW, among children with biopsy-proven kidney disease.

\section{Subjects and Methods}

\section{Patients}

We retrospectively reviewed the birthweights, gestational ages and medical histories of all patients who underwent renal biopsies from 1995 to 2011 at the Departments of Pediatrics or Medicine, Niigata University Medical and Dental Hospital.

The diagnosis of FSGS was based on the presence of focal and segmental sclerosis lesions in the diagnostic biopsies. The criteria for the decision to biopsy were as follows: (a) continuous proteinuria of approximately $0.3 \mathrm{~g} /$ day; (b) continuous hematuria and proteinuria, or (c) steroid-resistant nephrotic range of proteinuria in which proteinuria did not disappear for more than 4 weeks despite a high dose $(2 \mathrm{mg} / \mathrm{kg})$ of prednisolone treatment. This study was approved by the local Ethics Boards, and all patients provided informed consent for using the excess renal biopsy tissue available after diagnostic examination for research purposes. The histological evaluation of FSGS was performed according to the classification described by D'Agati et al. [12].

According to the World Health Organization classification scheme for birthweight, the infants were divided into the following categories: appropriate for gestational age (normal birthweight, NBW) $>2,500$ g, LBW $<2,500$ g, very low birthweight $<1,500$ g, and extremely low birthweight $(\mathrm{ELBW})<1,000 \mathrm{~g}$. The infants were also classified according to their gestational age: gestational age into full term, gestational age $\geq 37$ weeks; preterm, gestational age 32 36 weeks; very preterm, gestational age $<32$ weeks.
Table 1. Incidence of LBW in biopsy-proven kidney diseases

\begin{tabular}{lrll}
\hline Diagnosis & Cases & $\begin{array}{l}\text { LBW, } \\
\%\end{array}$ & $\begin{array}{l}\text { Premature, } \\
\%\end{array}$ \\
\hline IgA nephropathy & 86 & $3(3.5)$ & $1(1.1)$ \\
MCNS & 40 & $5(12.5)$ & $2(5.0)$ \\
HSPN & 25 & $1(4.0)$ & $0(0)$ \\
FSGS & 16 & $6(37.5)$ & $6(37.5)$ \\
Lupus nephritis & 14 & $1(7.1)$ & $1(7.1)$ \\
MPGN & 11 & $1(9.1)$ & $0(0)$ \\
Alport syndrome & 10 & $0(0)$ & $0(0)$ \\
Mesangial proliferative GN & 9 & $0(0)$ & $0(0)$ \\
Acute GN & 6 & $0(0)$ & $0(0)$ \\
Membranous nephropathy & 4 & $0(0)$ & $0(0)$ \\
Others & 22 & $2(9.1)$ & $0(0)$ \\
Total & 242 & $19(7.9)$ & $10(4.1)$ \\
\hline
\end{tabular}

HSPN = Henoch-Schenlein purpura nephritis; MPGN = membranoproliferative glomerulonephritis; $\mathrm{GN}$ = glomerulonephritis.

A total of 242 patients were diagnosed with kidney diseases with or without glomerular alterations, of which 16 were diagnosed with FSGS and enrolled in this study (table 1). Of these, 6 patients $(37.5 \%)$ were classified as LBW infants at birth. This rate was significantly higher than the mean LBW incidence in Japan (9.7\% in 2009).

Biopsy specimens from the 16 patients with FSGS were examined, and the morphological appearance was compared between specimens from NBW children $(\mathrm{n}=10)$ and LBW children $(\mathrm{n}=6)$ with FSGS. Twelve biopsy specimens from age-matched minimal change nephrotic syndrome (MCNS) patients with NBW were also compared. All MCNS patients underwent biopsies because of their unfavorable clinical course characterized by a significantly high frequency of relapse or high dependency on steroid treatment. Biopsies were performed before the administration of immunosuppressants and during the remission phase induced by steroid treatment.

Serum albumin and creatinine and 24-hour protein excretion levels were determined at the time of biopsy; the assays were performed by the Department of Biochemistry, Niigata University Medical and Dental Hospital. Medical records of body length and bodyweight that were taken at the time of blood tests were used for calculating the estimated glomerular filtration rate (eGFR). The eGFR values were calculated using the following Schwartz formula: height $(\mathrm{cm}) \times \mathrm{k} /$ serum creatinine value $(\mathrm{mg} / \mathrm{dl})$, where $\mathrm{k}=0.45$ for children $<2$ years old, 0.55 for children aged $2-12$ years, and 0.55 and 0.70 for female and male subjects aged $>12$ years, respectively [13].

\section{Quantification of Histological Damage in Renal Biopsies}

For light microscopy, renal specimens were taken by standard needle biopsy methods, fixed in Carnoy's solution and embedded in paraffin. Thereafter, 20 serial sections were cut at a thickness of $2 \mu \mathrm{m}$, and stained with periodic acid-Schiff (PAS) and periodic acid-methenamine silver (PAM). The specimens were reviewed 
and analyzed by an independent histopathologist who was blinded to the clinical data.

The morphometric analysis was performed using the SigmaScan Pro version 5.0 (Systat Software, Inc., Chicago, Ill., USA) and consisted of the following steps: (a) images of the PAM-stained fullsized glomerular cross-sections (GCS), which included the vascular pole, were captured and stored in a computer; (b) the outline of the glomerular tuft was traced to obtain the total glomerular area; (c) the number of nuclei in the mesangial, endocapillary, and extracapillary areas in each glomerulus was counted simultaneously and expressed as the mean number of glomerular nuclei per GCS or the mean number of glomerular nuclei per square millimeter; thus, both the actual number per GCS and the cell density per square millimeter of glomerular area were calculated. All cell counts were restricted to the glomerular tuft. To determine the optical number of glomerular sections, all glomeruli that included the vascular pole were examined in 2 tissue sections, which were separated by at least $30 \mu \mathrm{m}$ (equivalent to the thickness of 15 sections), and at least 12 different GCS were counted in each patient and expressed as median and interquartile range (25th75 th percentile). Globally sclerotic glomeruli were not included in the analysis. For podocyte counting, we assumed that each cell had only 1 nucleus and that nuclei were the same size. To prevent bias caused by enlarged nuclei in the pathologic glomeruli, the major axis of the nuclei was measured and compared between the groups.

For podocyte counting, the number density of podocytes $(\mathrm{Nv})$ or number of Wilms' tumor-1 (WT1)-positive cells was estimated using the Weibel and Gomez method [14]. Nv was estimated using this formula:

$$
\mathrm{Nv}=\mathrm{K} / \beta_{1} \times\left[\left(\mathrm{N}_{\mathrm{A}}\right)^{3} / \mathrm{V}_{\mathrm{V}}\right]^{1 / 2}
$$

where $\mathrm{K}$ is a size distribution coefficient that varies from 1 to 1.05 and can, therefore, be neglected; $\beta_{1}$ is the shape coefficient of the nuclei (1.55, the value for an ellipsoid); $\mathrm{N}_{\mathrm{A}}$ is the number of cell nuclei per $\mu \mathrm{m}^{2}$ of tuft profile area determined by image analysis described above, and $V_{V}$ is the volume fraction of cell nuclei in the glomerular tuft. To calculate the $\mathrm{V}_{\mathrm{V}}$ of cell nuclei in the glomerular tuft, a grid of coarse and fine points was superimposed over each glomerular image using the layers function, and the numbers of fine grid points hitting cell nuclear profiles and coarse grid points hitting the glomerular profile were counted. The volume fraction $\left(\mathrm{V}_{\mathrm{V}}\right)$ of cell nuclei in the glomerular tuft was calculated using the following formula:

$$
\mathrm{V}_{\mathrm{V}}=\mathrm{P}_{\text {cell }} /\left(\mathrm{P}_{\mathrm{gl}} \times \mathrm{X}\right)
$$

where $\mathrm{P}_{\text {cell }}$ is fine points hitting the cell number; $\mathrm{P}_{\mathrm{gl}}$ is the number of coarse grid points hitting the glomerular profile, and $\mathrm{X}$ is the ratio of coarse to fine points. The number of podocytes per glomerulus $(\mathrm{N})$ was then calculated using the following equation:

$$
\mathrm{N}=\mathrm{Nv} \times \mathrm{V}_{\mathrm{gl}}
$$

where $\mathrm{V}_{\mathrm{gl}}$ is glomerular volume and calculated using the formula:

$$
\mathrm{V}_{\mathrm{gl}}=(\text { mean glomerular tuft profile area })^{3 / 2} \times \beta_{2}
$$

where $\beta_{2}$ is the shape coefficient of glomeruli (1.38, the value for a sphere).

FSGS in Low Birthweight and Premature Birth Children

\section{Immunohistochemistry}

Antibodies used in this study was anti-WT49; an anti human WT1 protein (mouse monoclonal; Novocastra, Newcastle, UK), which was used for the primary antibody to detect glomerular podocytes. The secondary antibodies used included horseradish peroxidase-conjugated goat anti-mouse IgG (Dako Denmark A/S) and peroxidase-conjugated mouse antiperoxidase complexes (PAP; Dako Denmark A/S).

The detection of WT1-positive cells (podocytes) in 2- $\mu \mathrm{m}$ sections fixed in Carnoy's solution involved a 3-layer PAP method. The sections were dewaxed, treated, autoclaved for $20 \mathrm{~min}$ in $0.1 \mathrm{M}$ sodium citrate buffer ( $\mathrm{pH}$ 9.0; Target Retrieval Solution; Dako Denmark A/S), and washed in phosphate-buffered saline (PBS). The sections were then blocked in $10 \%$ normal goat serum, $10 \%$ fetal calf serum, and $1 \%$ bovine serum albumin (BSA) in PBS for $30 \mathrm{~min}$, followed by a second blocking step in 10\% BSA in PBS for $15 \mathrm{~min}$ at room temperature. Primary antibodies that were diluted in $10 \%$ normal human serum and $1 \%$ BSA in PBS were added to the sections and incubated overnight at $4^{\circ} \mathrm{C}$. Sections were then washed 3 times in PBS; endogenous peroxidase was inactivated in $0.3 \% \mathrm{H}_{2} \mathrm{O}_{2}$ in methanol for $20 \mathrm{~min}$ and washed again in PBS. After washing in PBS, sections were incubated with horseradish peroxidase-conjugated secondary antibodies, followed by PAP complexes, for $45 \mathrm{~min}$ each at room temperature. After another washing with PBS, sections were developed with a diaminobenzidine substrate (Dako Denmark A/S). PAS counterstaining was then performed in order to stain the basement membrane. The number of WT1-positive cells was counted in at least 12 GCS per patient in the same manner as in the light microscopic assessments using PAM-staining sections.

\section{Statistical Analyses}

One-way analysis of variance with post hoc analysis using Tukey's multiple-comparison test was used for comparisons between the 3 groups. The Brown-Forsythe test was used to test if samples are from populations with equal variances. In the samples with defect variances, the Kruskal-Wallis test with post hoc analysis using Dunn's multiple-comparison test was used for comparisons between the 3 groups (GraphPad 6.0, GraphPad Software, Inc., La Jolla, Calif., USA). Data are expressed as median and interquartile range ( 25 th- 75 th percentile), and $p$ values $<0.05$ were considered statistically significant.

\section{Results}

\section{Clinical Profile}

The clinical characteristics of each patient group at biopsy are summarized in table 2. Most cases of FSGS were detected by the annual urinary screening program in school or preschool children aged $5-15$ years (12 patients; $75.0 \%)$, while the other childhood cases were detected by chance during urinary investigations (4 patients; $25.0 \%$ ). Particularly, all patients in the LBW-FSGS group were identified through the annual urinary screening program. In contrast, most of the patients in the MCNS group were discovered mainly due to symptoms of general edema, 
Table 2. Comparison of clinical parameters at biopsy

\begin{tabular}{lccc}
\hline & MCNS & NBW-FSGS & LBW-FSGS \\
\hline Patients & 12 & 10 & 6 \\
Males:females & $8: 4$ & $7: 3$ & $3: 3$ \\
Age at biopsy, years & $13.9(10.1-16.4)$ & $11.4(8.8-13.0)$ & $13.6(10.8-15.0)$ \\
Body height, cm & $152.5(133.5-156.6)$ & $147.1(131.3-155.8)$ & $150.4(133.7-173.7)$ \\
Body height (percentile) & $15.0(10.0-24.5)$ & $56.5(38.5-72.3)$ & $62.0(9.0-93.0)$ \\
Body weight, kg & $46.4(31.3-53.6)$ & $45.0(30.0-49.9)$ & $37.5(25.9-60.0)$ \\
Body weight (percentile) & $45.5(6.3-62.3)$ & $66.5(15.8-80.0)$ & $5.0(2.0-43.0)$ \\
Serum albumin, g/dl & $3.7(3.3-4.3)$ & $3.9(3.1-4.5)$ & $3.7(3.5-4.4)$ \\
Serum creatine, mg/dl & $0.50(0.41-0.60)$ & $0.40(0.36-0.54)$ & $0.70(0.52-1.11)^{*}$ \\
eGFR, ml/min/1.73 m ${ }^{2}$ & $111.8(103.8-121.4)$ & $126.3(115.1-140.2)$ & $90.2(57.9-103.5)^{\mathrm{b}, * * *}$ \\
Proteinuria, g/day & $0.04(0.01-0.15)$ & $1.12(0.43-1.50)^{\mathrm{b}}$ & $1.30(0.51-1.91)^{\mathrm{b}}$ \\
Birthweight, g & $3,110(2,860-3,420)$ & $3,315(2,983-3,492)$ & $917(752-1,078)^{\mathrm{a}, * * *}$ \\
Gestational age, week & $39.0(39.0-40.0)$ & $40.0(39.0-40.0)$ & $25.5(23.0-27.5)^{\mathrm{b}, * * *}$ \\
\hline
\end{tabular}

Kruskal-Wallis test with post hoc analysis using Dunn's multiple-comparison test was used for the analyses of serum creatinie and gestational age; otherwise, one-way analysis of variance with post hoc analysis using Tukey's multiple comparison test was used for comparisons between the 3 groups. Data are expressed as median and interquartile range (25th-75th percentile).

${ }^{\mathrm{a}} \mathrm{p}<0.001,{ }^{\mathrm{b}} \mathrm{p}<0.01$ vs. MCNS group; ${ }^{* * *} \mathrm{p}<0.001,{ }^{*} \mathrm{p}<0.05$ vs. NBW-FSGS group.

and they underwent biopsies because of their unfavorable clinical course, which was often characterized by a significantly high frequency of relapse or steroid dependency. Biopsies for patients in the MCNS group were performed before the administration of immunosuppressants and during the remission phase induced by steroid treatment.

The degree of proteinuria at discovery was significantly higher in the LBW-FSGS group than in the NBWFSGS group. However, no difference in serum albumin level was detected among the groups (table 2). Kidney function (eGFR) at the time of the biopsy was significantly reduced in the LBW-FSGS group than in the MCNS or NBW-FSGS groups. In the LBW-FSGS group, 5 out of 6 children were born ELBW $(<1,000 \mathrm{~g})$, with the exception of 1 patient whose birthweight was $1,078 \mathrm{~g}$. Similarly, 5 out of 6 children were born preterm, with a gestational age of $<32$ weeks, with the exception of 1 patient whose gestational age was 32 weeks; however, all of the LBW patients were appropriate for dates according to the data reported by the Japanese Society of Pediatrics in 2010 (table 2).

\section{Renal Histology}

Prominent glomerular hypertrophy with focal and segmental matrix expansion was the most common finding in the biopsies of patients from the LBW-FSGS group. According to the classification proposed by D'Agati et al. [12], histological variants observed in the LBW-FSGS group were a perihilar variant in 3 out of $6(50 \%)$ patients, which was characterized by perihilar sclerosis in more than $50 \%$ of glomeruli with segmental lesions and at least 1 glomerulus with perihilar hyalinosis (fig. 1). Although perihilar hyalinosis was not detected in the other 3 patients, their glomerular findings were consistent with a perihilar variant with segmental perihilar sclerosis. By contrast, focal and segmental cell proliferation and matrix expansion were the only findings in patients of the NBWFSGS group, and the histological assessment revealed no distinctive lesions; therefore, this was categorized as FSGS not otherwise specified. The glomerular size was almost identical to that in patients of the MCNS group (fig. 1; table 3). The number of glomeruli showing segmental or global glomerular sclerosis was significantly greater in the LBW-FSGS group than in the NBW-FSGS group (table 3 ).

The quantification of glomerular cellularity revealed significant reductions in podocyte number and podocyte density in both the NBW-FSGS and LBW-FSGS groups as compared to the values in the MCNS group (table 3 ). To exclude potential bias introduced by the enlarged glomerular size resulting in spurious reduction of podocyte number, podocyte density was adjusted to glomerular size. Even after adjusting for glomerular size, the number of podocytes in the LBW-FSGS group was $48 \%$ lower than that in the MCNS group and 33\% lower than that in the NBW-FSGS group. NBW-FSGS patients showed $23 \%$ fewer podocytes than the MCNS group (fig. 2). Cell den- 

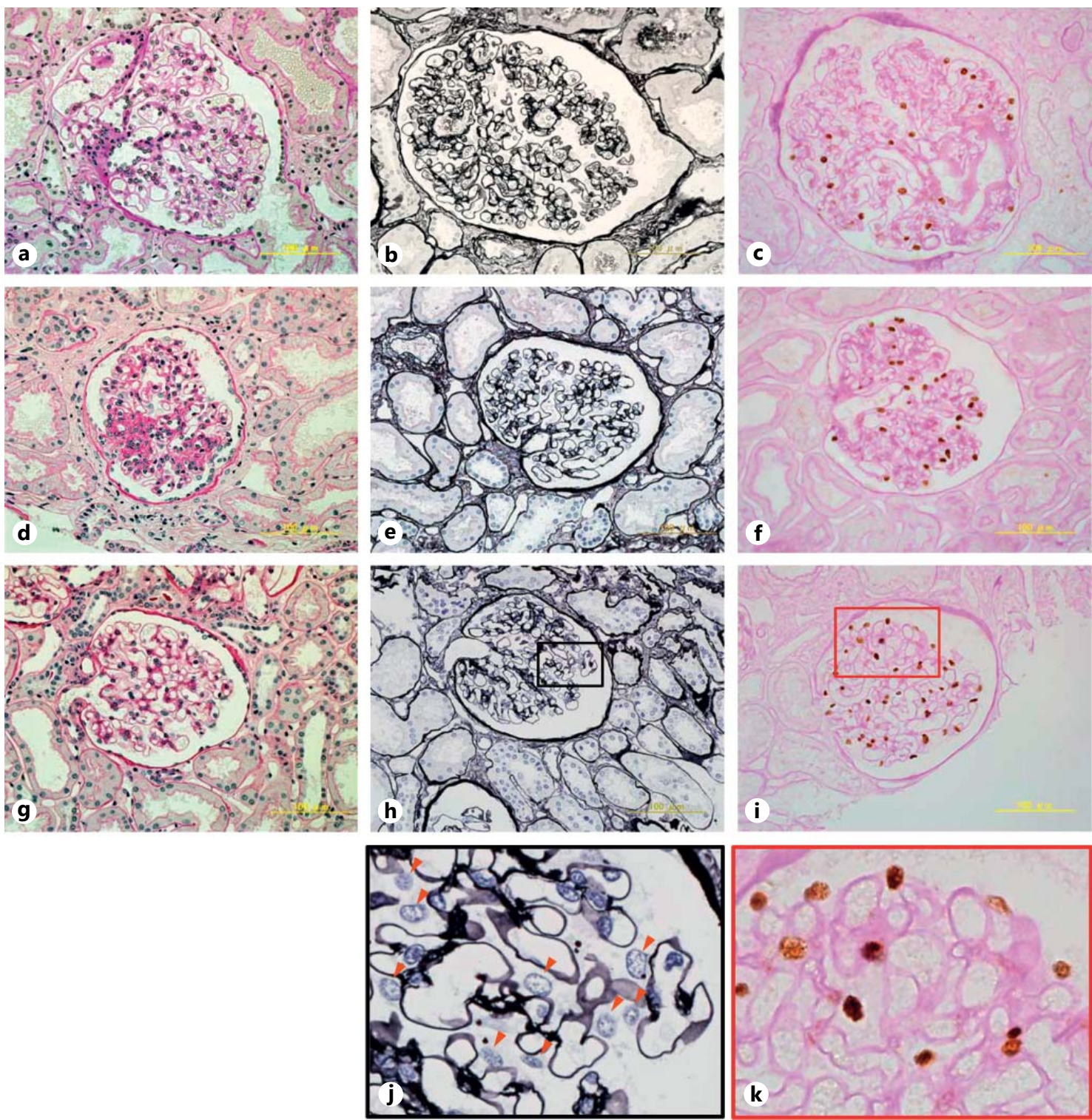

Fig. 1. Photomicrographs of biopsy specimens in the LBW-FSGS (a-c), NBW-FSGS (d-f), and MCNS (g-k) groups. a, d, g PAS staining. b, e, h, j PAM silver staining. c, f, i, k Immunohistochemistry for WT1 protein with PAS counter staining. $\mathbf{j}$ Red arrowheads indicate extracapillary cells (podocytes). $\mathbf{k}$ Nuclei of WT1-positive cells are shown in brown color. Original magnification, $\times 400$.

sity in the mesangial area was significantly lower in the LBW-FSGS group than in the NBW-FSGS group ( $\mathrm{p}<$ 0.05 ), whereas no difference in endocapillary cell density was detected between the LBW-FSGS and the MCNS group. However, when adjusting these cell numbers for glomerular size, the numbers of total glomerular cells, endocapillary cells, and cells in the mesangial area were significantly higher in both the NBW-FSGS and LBWFSGS groups than in the MCNS group; however, the cor- responding number in the LBW-FSGS group was much higher than that in the NBW-FSGS group (table 3; fig. 2).

Immunohistochemistry for WT1-positive cells revealed almost similar results that obtained by the method using PAM-stained sections in the analyses among the groups (table 3; fig. 2). Although the nuclei of extracapillary cells or WT1 positive cells tended to be enlarged in the LBW-FSGS group, there was no statistically significant difference between the groups (fig. 2). 
Table 3. Comparison of histological findings

\begin{tabular}{llccc}
\hline & & MCNS & NBW-FSGS & LBW-FSGS \\
\hline Patients & & 12 & 10 & 6 \\
\hline Glomeruli with sclerotic lesion & $\%$ & 0 & $6.7(5.7-10.8)^{\mathrm{b}}$ & $16.7(12.5-29.2)^{\mathrm{a}}$ \\
\hline Glomerular size & $\mu^{2}$ & $22,052(17,909-23,737)$ & $23,644(21,916-27,430)$ & $34,599(33,513-43,903)^{\mathrm{a}, * * *}$ \\
\hline Total glomerular cell number & $/ \mathrm{GCS}$ & $117.3(112.5-121.6)$ & $135.8(122.5-143.6)^{\mathrm{a}}$ & $168.3(143.8-174.3)^{\mathrm{a}, * *}$ \\
& $/ \mathrm{mm}^{2}$ & $4,588(4,035-5,864)$ & $6,405(5,702-6,668)$ & $5,860(5,363-6,372)^{\mathrm{c}, * *}$ \\
\hline Endocapillary cell number & $/ \mathrm{GCS}$ & $39.4(36.9-40.5)$ & $52.5(46.0-60.4)^{\mathrm{a}}$ & $76.6(70.0-79.5)^{\mathrm{a}, * * *}$ \\
& $/ \mathrm{mm}^{2}$ & $2,182(1,791-2,794)$ & $2,335(1,976-2,785)^{\mathrm{c}}$ & $1,965(1,778-2,108)$ \\
\hline Cells in mesangial area & $/ G C S$ & $39.7(37.4-41.6)$ & $50.7(45.2-54.3)^{\mathrm{b}}$ & $69.6(54.4-71.6)^{\mathrm{a}, * * *}$ \\
& $/ \mathrm{mm}^{2}$ & $2,031(1,623-2,267)$ & $2,309(2,100-2,704)^{\mathrm{c}}$ & $2,010(1,836-2,175)^{*}$ \\
\hline Podocyte number & $/ \mathrm{GCS}^{2}$ & $39.6(33.1-41.0)$ & $36.0(30.6-38.2)$ & $21.4(19.4-24.1)^{\mathrm{a}, * * *}$ \\
& $/ \mathrm{mm}^{2}$ & $1,900(1,771-2,269)$ & $1,352(1,227-1,449)^{\mathrm{a}}$ & $608(443-729)^{\mathrm{a}, * * *}$ \\
\hline WT1+ cell number & $/ \mathrm{GCS}^{2}$ & $36.3(31.5-41.4)$ & $31.4(28.5-36.5)$ & $22.0(20.0-24.9)^{\mathrm{a}, * *}$ \\
& $/ \mathrm{mm}^{2}$ & $2,171(1,892-2,329)$ & $1,505(1,319-1,684)^{\mathrm{a}}$ & $678(493-792)^{\mathrm{a}, * * *}$ \\
\hline
\end{tabular}

Kruskal-Wallis test with post hoc analysis using Dunn's multiple comparison test was used for the analyses of glomeruli with sclerotic lesion; otherwise, one-way analysis of variance with post hoc analysis using Tukey's multiple comparison test was used for comparisons between the 3 groups. Data are expressed as median and interquartile range (25th-75th percentile). ${ }^{\mathrm{a}} \mathrm{p}<0.001,{ }^{\mathrm{b}} \mathrm{p}<0.01$, ${ }^{c} \mathrm{p}<0.05$ vs. MCNS group; ${ }^{* * *} \mathrm{p}<0.001,{ }^{* *} \mathrm{p}<0.01,{ }^{*} \mathrm{p}<0.05$ vs. NBW-FSGS group.

The number of podocytes calculated by the Weibel and Gomez method [14] revealed that the number of podocytes per glomerulus in the LBW-FSGS group was 34\% lower than that in the MCNS group and 24\% lower than that in the NBW-FSGS group (fig. 2). Moreover, a similar result was obtained in the number of WT1-positive cells per glomerulus (fig. 2).

\section{Discussion}

Recent epidemiologic studies that have examined the association between LBW and kidney function have reported a significant association between LBW and endstage kidney disease $[4,15]$, suggesting that LBW could be a risk factor for progressive kidney disease in later life. However, the underlying pathogenesis has not been fully elucidated. Our retrospective study revealed that the incidence of LBW among children diagnosed with FSGS was surprisingly high (37.5\%). We found that the number of podocytes in LBW children with FSGS was significantly lower than that in MCNS children or NBW children with FSGS. In addition, most of these LBW children had ELBW associated with very premature gestational age, indicating that not only LBW but also prematurity could be a risk factor for podocytopenia and FSGS. Indeed, the fact that all of the LBW children examined in this study were appropriate for dates supported our hypothesis that glomerular structure prematurity might be a more important factor than direct podocyte injury during the fetal period. This association between LBW and glomerular structure prematurity can be mediated by many factors, including stress, poor nutrition, drug addiction, alcohol abuse, and heart disease or hypertension in the expectant mother.

It could be argued that our methodology for counting podocytes was not adequate since the Weibel-Gomez method that we used in the present study often overestimates the glomerular cell number [16]. The dissector/ fractionator method is currently considered the theoretic gold standard method for podocyte number estimation in which the number of cells is counted in a fraction of sections and is free of bias $[16,17]$. However, the dissector/ fractionator method requires exhaustive serial sectioning of the glomerulus because of the limited amount of tissue available for histological assessments other than for diagnostic purposes. In the current study, we initially measured cell density to avoid the bias introduced by counting cells in different glomerular sections with different 


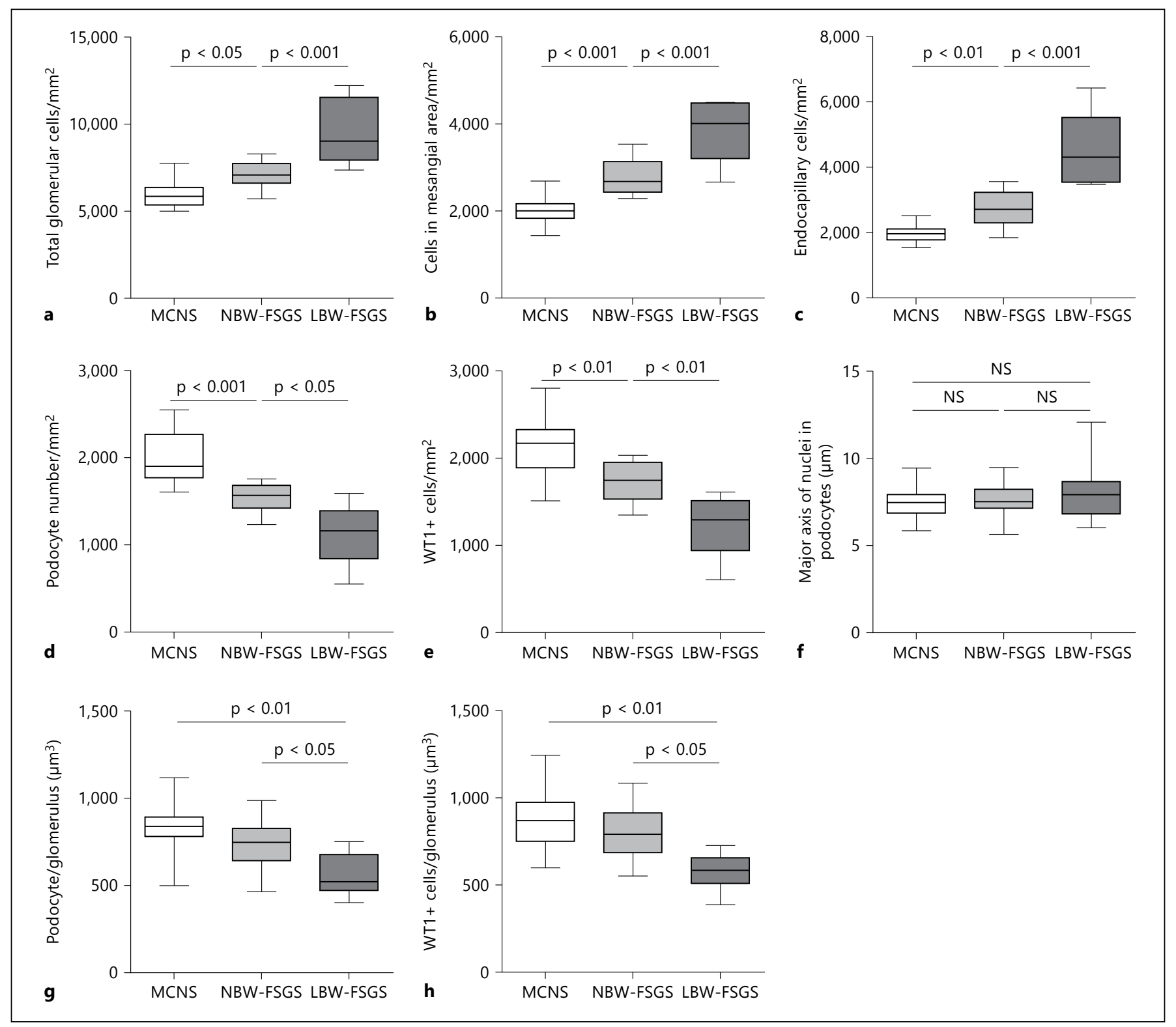

Fig. 2. Summary of histological findings in LBW-FSGS and NBWFSGS children in comparison to the MCNS group. a Glomerular cellularity (total nuclei within the glomerular tuft). b Cells in the mesangial area. c Endocapillary cellularity. d Podocytes. e WT1positive cells. $\mathbf{f}$ Major axis of nuclei in podocytes. $\mathbf{g}$ Number of podocytes per glomerulus. $\mathbf{h}$ Number of WT1-positive cells per glomerulus. Cells or nuclei were assessed in full-sized GCS including the vascular pole, and the mean number of glomerular nuclei per square millimeter, which reflects the cell density per glomerulus was calculated. a-e Furthermore, cell densities were adjusted for capillary size. $\mathbf{g}, \mathbf{h}$ The number of cells per glomerulus was calculated using the method of Weibel and Gomez (see text for details). The box plot extends from the 25 th percentile to the 75 th percentile, with the horizontal line at the median, and the whiskers indicate range. areas, and compared cell density by adjusting the values to glomerular size to avoid the potential effects of increased glomerular area cell number. The results revealed a similar tendency for a reduction in the number of podocytes between the Weibel-Gomez method and our meth- od. Therefore, although our counting methodology may not yield the actual number of cells, it is appropriate for comparing the differences among the groups.

LBW or premature birth, however, is not always associated with the loss of kidney function or the develop- 
ment of FSGS. Our findings showed that the occurrence of glomerulomegaly was not correlated with the focal sclerosis; moreover, half of our patients had no history of LBW or premature birth, suggesting the presence of a second trigger that may have initiated or promoted the podocyte loss. The low nephron number caused by the LBW may have influenced the vulnerability of the glomerular capillary wall as the first trigger and modified the course of a subsequent renal injury. Indeed, our study revealed that LBW or premature birth was associated with worse outcomes in FSGS (table 2).

The association observed between MCNS and LBW is worthy of discussion. Most of the patients with MCNS underwent biopsies because of their unfavorable clinical course, such as the significantly high frequency of relapse or high dependency on steroid treatment. Thus, the relatively high incidence of LBW among the MCNS group in our data (12.5\%), along with the findings reported in other studies, suggested that LBW could also be a potential risk factor for refractory nephrotic syndrome [18-20]. It is also noteworthy that most of the patients in the LBWFSGS group were born ELBW $(<1,000 \mathrm{~g})$, while the mean birthweight and gestational age of LBW children in the MCNS group were 2,307.2 $\pm 260.2 \mathrm{~g}$ and $36.2 \pm 2.6$ weeks, respectively. There were no histological differences between the NBW-MCNS and LBW-MCNS groups, including the podocyte numbers (data not shown). The finding that only 2 out of 5 patients in the LBW-MCNS group were born prematurely whereas all patients in the LBW-FSGS were premature (table 1) suggested that prematurity rather than LBW is a significant risk factor for podocyte loss and FSGS lesions. LBW is the result of either preterm birth or intrauterine growth retardation, wherein birthweight may be low but appropriate for gestational age. It has been reported that in some developed countries, including Japan, the incidence of premature and LBW infants has markedly increased in the past few decades $[21,22]$. In Japan, the premature birth rate rose from 4.2 to $5.7 \%$ between 1980 and 2009, while the LBW rate rose from 5.1 to $9.7 \%$ between 1975 and 2010 [23]. Further, recent studies have revealed that the frequency of FSGS among biopsies in both children and adults is increasing in certain countries [24-27]. Although there is no evidence currently suggesting that the increasing incidence of LBW is associated with increasing FSGS occurrence, the inferences are enough to cause concern, particularly in countries with an increasing incidence of LBW.

Although the cause of this phenomenon has not been determined, recent epidemiological studies have provid-

ed certain clues. The increasing incidence of obesity among children and adolescents worldwide could be a potential factor contributing to the rise in FSGS frequency, which is probably secondary to the glomerular hyperfiltration that occurs in obese subjects [28]. In addition, there are increasing lines of evidence that LBW is associated with obesity [29]. Several studies have described that glomerular hyperfiltration occurs in obese subjects or subjects with a history of LBW, in whom the nephron number may be reduced. These glomeruli that adapt through hypertrophy and hyperfiltration in order to maintain renal function would be exposed to mechanical stretching of the capillary wall that could cause the detachment of podocytes from the basement membrane, which may finally lead to progressive glomerulosclerosis and a decline in renal function [30-32]. This hypothesis is supported by our findings that most of the patients in the LBW-FSGS group had FSGS lesions of the perihilar variant, which is common in secondary forms of FSGS and is mediated by an adaptive response to nephron loss or glomerular hypertension [12].

In summary, our findings showed that LBW and premature birth were associated with the development of FSGS. The possibility that LBW predisposes to a prematurity of the glomerular structure and more severe podocyte loss in children with FSGS warrants further investigation.

\section{Acknowledgements}

This work was supported by Grants-in-Aid for Scientific Research (16790569 to Y.I. and 21390307 to H.N.) from the Ministry of Education, Culture, Sports, Science and Technology of Japan, and Grants-in-Aid for Promotion of Niigata University Research Projects from Niigata University.

References

Am J Nephrol 2013;38:149-157 DOI: $10.1159 / 000353898$
Ikezumi/Suzuki/Karasawa/Yamada/

Hasegawa/Nishimura/Uchiyama 
$\checkmark 4$ White SL, Perkovic V, Cass A, Poulter NR, Spector T, Haysom L, Craig JC, Salmi IA, Chadban SJ, Huxley RR: Is low birth weight an antecedent of CKD in later life? A systematic review of observational studies. Am J Kidney Dis 2009;54:248-261.

5 Luyckx VA, Brenner BM: Low birth weight, nephron number, and kidney disease. Kidney Int Suppl 2005;97:S68-S77.

-6 Bertram JF, Douglas-Denton RN, Diouf B, Hughson MD, Hoy WE: Human nephron number: implications for health and disease. Pediatr Nephrol 2011;26:1529-1533.

7 Hodgin JB, Rasoulpour M, Markowitz GS, D’Agati VD: Very low birth weight is a risk factor for secondary focal segmental glomerulosclerosis. Clin J Am Soc Nephrol 2009;4:71-76.

$\checkmark 8$ Matsusaka T, Xin J, Niwa S, Kobayashi K, Akatsuka A, Hashizume H, Wang QC, Pastan I, Fogo AB, Ichikawa I: Genetic engineering of glomerular sclerosis in the mouse via control of onset and severity of podocyte-specific injury. J Am Soc Nephrol 2005;16:1013-1023.

-9 Laurens WE, Vanrenterghem YF, Steels PS, Van Damme BJ: A new single nephron model of focal and segmental glomerulosclerosis in the Munich-Wistar rat. Kidney Int 1994;45: 143-149.

10 Wharram BL, Goyal M, Wiggins JE, Sanden SK, Hussain S, Filipiak WE, Saunders TL, Dysko RC, Kohno K, Holzman LB, Wiggins RC: Podocyte depletion causes glomerulosclerosis: diphtheria toxin-induced podocyte depletion in rats expressing human diphtheria toxin receptor transgene. J Am Soc Nephrol 2005;16:2941-2952.

-11 D’Agati VD, Kaskel FJ, Falk RJ: Focal segmental glomerulosclerosis. N Engl J Med 2011; 365:2398-2411.

-12 D’Agati VD, Fogo AB, Bruijn JA, Jennette JC: Pathologic classification of focal segmental glomerulosclerosis: a working proposal. Am J Kidney Dis 2004;43:368-382.
13 Schwartz GJ, Brion LP, Spitzer A: The use of plasma creatinine concentration for estimating glomerular filtration rate in infants, children, and adolescents. Pediatr Clin North Am 1987;34:571-590.

14 Weibel ER, Gomez DM: A principle for counting tissue structures on random sections. J Appl Physiol 1962;17:343-348.

-15 Vikse BE, Irgens LM, Leivestad T, Hallan S, Iversen BM: Low birth weight increases risk for end-stage renal disease. J Am Soc Nephrol 2008; 19:151-157.

16 White KE, Bilous RW: Estimation of podocyte number: a comparison of methods. Kidney Int 2004;66:663-667.

17 Nyengaard JR: Stereologic methods and their application in kidney research. J Am Soc Nephrol 1999;10:1100-1123.

18 Teeninga N, Schreuder MF, Bökenkamp A, Delemarre-van de Waal HA, van Wijk JA: Influence of low birth weight on minimal change nephrotic syndrome in children, including a meta-analysis. Nephrol Dial Transplant 2008;23:1615-1620.

19 Sheu JN, Chen JH: Minimal change nephrotic syndrome in children with intrauterine growth retardation. Am J Kidney Dis 2001;37: 909-914.

20 Zidar N, Avgustin Cavić M, Kenda RB, Ferluga D: Unfavorable course of minimal change nephrotic syndrome in children with intrauterine growth retardation. Kidney Int 1998; 54:1320-1323.

21 Hamilton BE, Miniño AM, Martin JA, Kochanek KD, Strobino DM, Guyer B: Annual summary of vital statistics: 2005. Pediatrics 2007;119:345-360.

22 Takimoto H, Yokoyama T, Yoshiike N, Fukuoka H: Increase in low-birth-weight infants in Japan and associated risk factors, 19802000. J Obstet Gynaecol Res 2005;31:314322.
23 Ministry of Health, Labour and Welfare, Japan: Vital statistics in Japan - the latest trends. http://www.mhlw.go.jp/english/database/dbhw/vs01.html (accessed Jan 15, 2013).

24 Banaszak B, Banaszak P: The increasing incidence of initial steroid resistance in childhood nephrotic syndrome. Pediatr Nephrol 2012; 27:927-932.

25 Borges FF, Shiraichi L, da Silva MP, Nishimoto EI, Nogueira PC: Is focal segmental glomerulosclerosis increasing in patients with nephrotic syndrome? Pediatr Nephrol 2007; 22:1309-1313.

26 Swaminathan S, Leung N, Lager DJ, Melton LJ 3rd, Bergstralh EJ, Rohlinger A, Fervenza FC: Changing incidence of glomerular disease in Olmsted County, Minnesota: a 30-year renal biopsy study. Clin J Am Soc Nephrol 2006; 1:483-487.

27 Kitiyakara C, Kopp JB, Eggers P: Trends in the epidemiology of focal segmental glomerulosclerosis. Semin Nephrol 2003;23:172-182.

28 Srivastava T: Nondiabetic consequences of obesity on kidney. Pediatr Nephrol 2006;21: 463-470.

29 Morrison JL, Duffield JA, Muhlhausler BS, Gentili S, McMillen IC: Fetal growth restriction, catch-up growth and the early origins of insulin resistance and visceral obesity. Pediatr Nephrol 2010;25:669-677.

30 Manalich R, Reyes L, Herrera M, Melendi C, Fundora I: Relationship between weight at birth and the number and size of renal glomeruli in humans: a histomorphometric study. Kidney Int 2000;58:770-773.

-31 Hinchliffe SA, Lynch MR, Sargent PH, Howard CV, Van Velzen D: The effect of intrauterine growth retardation on the development of renal nephrons. Br J Obstet Gynaecol 1992; 99:296-301.

- 32 Kriz W, Gretz N, Lemley KV: Progression of glomerular diseases: is the podocyte the culprit? Kidney Int 1998;54:687-697. 